
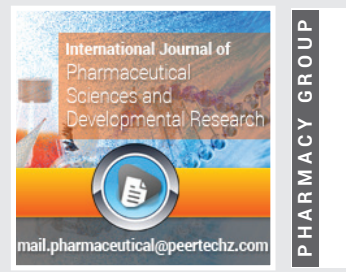

Tomomi Hatanaka, ${ }^{1,2, \#, ~ S a k i ~}$

Yamamoto', Mayuko Kamei', Wesam

R Kadhum' ', Hiroaki Todo' and Kenji

Sugibayashi ${ }^{* *}$,\#

'Faculty of Pharmaceutical Sciences, Josai University, Sakado, 350-0295, Japan

${ }^{2}$ Tokai University School of Medicine, Isehara, 2591193, Japan

\#These authors contributed equally to this work.

Dates: Received: 08 December, 2016; Accepted: 20 January, 2017; Published: 23 January, 2017

*Corresponding author: Kenji Sugibayashi, Professor, Faculty of Pharmaceutical Sciences, Josai University, Keyakidai 1-1, Sakado, Saitama 3500295, Japan, Tel/Fax: +81-492-71-7943; E-mail: sugib@josai.ac.jp

Keywords: Skin concentration; Dermally metabolized chemical; In silico estimation; Skin permeation; Skin metabolism; Ester compound

https://www.peertechz.com

\section{Research Article \\ In Silico Estimation of Skin Concentration of Dermally Metabolized Chemicals}

\section{Abstract}

Background: A great deal of in silico estimation methods were proposed for skin concentration and permeation of drugs by many researchers including us. The aim of the present study was to expand our in silico estimation method of skin concentration to dermally metabolized chemicals.

Materials and Methods: A three-layered diffusion model consisting of stratum corneum, viable epidermis and dermis was constructed based on Fick's second law of diffusion incorporated with MichaelisMenten equation and plasma clearance in the viable epidermis and dermis, respectively. Ethyl nicotinate was used as a model chemical, and the in vivo skin concentration of the ester and its metabolite, nicotinic acid were measured after topical application to hairless rats. Permeation parameters were determined from the in vitro permeation data through full-thickness skin and stripped skin after application of the ester or acid with and without esterase inhibitor treatment. Metabolic parameters were obtained from the metabolic profile of the ester using skin homogenate.

Results and Conclusion: The skin concentrations calculated from our improved model using the permeation and metabolic parameters obtained beforehand were similar to the observed values. Influence of cutaneous enzyme distribution and plasma clearance on the skin concentrations were also estimated using appropriately modified models, resulting in higher influence on the acid than the ester. This estimation method will become an effective tool to assess the efficacy and safety of dermally metabolized chemicals.

\section{Abbreviations}

EN: Ethyl Nicotinate; NA: Nicotinic Acid; DPF: Diisopropyl Fluorophosphates

\section{Introduction}

Skin has been the administration site for therapeutic agents for skin diseases and disorders since ancient times [1]. Because skin is the direct interface with the environment, it also undergoes daily exposure to external substances including toxicants [2]. The pharmacological and toxicological activities of chemical compounds depend on their concentrations at the site of action, so many methods for estimating skin concentration have been developed by utilizing a variety of techniques, including tape-stripping [3], microdialysis [4], Raman spectrophotometry [5] and in silico modeling [6,7].

In silico modeling is a promising method for evaluating efficacy and safety after dermal exposure to topically active compounds. Once the modeling of the permeation process is completed, the skin concentration of chemicals can be estimated noninvasively, rapidly, and at a low cost. Mammalian skin is composed of two primary layers, the epidermis and subjacent dermis; the former consists of the stratum corneum, stratum granulosum, stratum spinosum and stratum basale [8]. The stratum corneum, outermost epidermal layer, consists of 1025 layers of dead, elongated, and fully keratinized corneocytes and the intercellular lipid lamellae linked to the cornified envelope of corneocytes, so that this layer is frequently described by "brick and mortar" model [9]. Because the lipid matrix in the stratum corneum greatly contributes to the skin's barrier function [10] and only a small area of skin surface is occupied by appendages such as hair follicles, sebaceous glands and sweat glands [9], the two-layered membrane consisting of the stratum corneum and its lower layers has been often assumed in in silico models. We previously demonstrated that the mean skin concentration and concentration at each depth in the skin layers can be estimated based on the two-layered diffusion model for drugs and chemicals with a wide range of lipophilicity [11,12].

Although in silico modeling provides sufficiently satisfactory predictability of skin concentration, this is not the case for compounds that are easily metabolized in the skin. These 
compounds applied via the stratum corneum diffuse through the viable epidermis, which is the most metabolically active layer in the skin and has many enzymes similar to the liver [13]. Biotransformation in the viable epidermis may change the biological activity of compounds after passage through this layer. In fact, most steroids are applied to the skin as antedrugs to avoid systematic adverse effects; they are metabolically inactivated in the skin as well as in the blood circulation [14]. On the other hand, there have been numerous attempts at transdermal drug delivery via the prodrug derivation [15]. After having overcome the first critical step in skin permeation, i.e. permeation via the stratum corneum, the prodrugs are metabolized to drugs prior to exhibiting their biological effects. Understanding of the skin concentrations of both the applied chemical compound and its metabolite is required to correctly evaluate the efficacy and safety of the drugs.

Although our previous estimation for skin concentration used in vitro data after permeation experiments using the excised skin, the final goal is to estimate the in vivo skin concentration. The papillary layer of dermis directly under the epidermis contains blood microcirculation consisting of permeable capillaries and thus the plasma clearance plays an important role in the determination of skin concentration after dermal application of compounds [16]. It is expected that the influence of plasma clearance makes the estimation of skin concentration of dermally metabolized chemicals more complicated: skin concentrations of both applied chemical and its metabolite will be dramatically changed by the combination of distribution of metabolic enzyme in skin and location of cutaneous microvasculature.

The aim of the present study was to expand our in silico estimation method of skin concentration to chemicals that are easily metabolized in the skin. A three-layered diffusion model consisting of stratum corneum, viable epidermis and dermis was constructed based on the Fick's second law of diffusion, with Michaelis-Menten equation and plasma clearance in the viable epidermis and dermis, respectively. Ethyl nicotinate (EN) was used as a model compound, because we have already confirmed that EN is metabolized to nicotinic acid (NA) in the human and rat skin $[17,18]$. The skin permeation experiments were carried out using excised hairless rat skin. The permeation parameters, such as the diffusion coefficient and partition coefficient, were determined from the amount permeated through full-thickness skin and stratum corneumstripped skin after application of the ester or metabolite with and without an esterase inhibitor. The metabolic parameters, i.e. the Michaelis constant and maximum metabolic rate in the Michaelis-Menten equation, were obtained from the hydrolysis profiles of the ester in a skin homogenate. The skin concentrations of the ester and metabolite were calculated based on our improved three-layered diffusion model, using the experimentally obtained permeation and metabolic parameters and the literature values of plasma clearance. The in vivo skin concentrations were separately measured $6 \mathrm{~h}$ after dermal application of the ester compound to hairless rat, and the observed values were compared with the calculated values. Influence of cutaneous enzyme distribution and plasma clearance on the skin concentration of the dermally metabolized chemical were also simulated using appropriately modified three-layered diffusion models.

\section{Materials and Methods}

\section{Chemicals and reagents}

EN and NA were purchased from Tokyo Chemical Industry Co., Ltd. (Tokyo, Japan). Diisopropyl fluorophosphate (DFP) was obtained from Wako Pure Chemical Industries, Ltd. (Osaka, Japan). Other chemicals and reagents were of special grade or HPLC grade obtained commercially and used without further purification.

\section{Animals}

Male hairless rats (WBN/Ila-Ht) weighing 200-250 g were obtained from the Life Science Research Center, Josai University (Sakado, Saitama, Japan) or Ishikawa Experimental Animal Laboratories (Fukaya, Saitama, Japan). All procedures for animal care and experimental protocols were reviewed and approved by the Institutional Animal Care and Use Committee of Josai University.

\section{Skin permeation experiments}

Abdominal skin of hairless rat was freshly excised under anesthesia by intraperitoneal injection of a combination anesthetic $(0.3 \mathrm{mg} / \mathrm{kg}$ of medetomidine, $4.0 \mathrm{mg} / \mathrm{kg}$ of midazolam, and $5.0 \mathrm{mg} / \mathrm{kg}$ of butorphanol) immediately before permeation. Stripped skin was prepared by stripping the stratum corneum 20 times with cellophane tape (Cellotape ${ }^{\mathrm{TM}}$, No. 405, Nichiban Co. Ltd., Tokyo, Japan) [19]. Excess subcutaneous fat was trimmed off from the excised full-thickness or stripped skin, and the obtained skin sample was mounted on a sideby-side diffusion cell (effective diffusion area of $0.95 \mathrm{~cm}^{2}$ ), in which the donor (epidermis side) and receiver (dermis side) chambers were prewarmed to $32^{\circ} \mathrm{C}$. The skin was hydrated by filling both chambers with $3.0 \mathrm{~mL}$ of phosphate buffered saline (PBS; $\mathrm{pH} 7.4$ ) for $30 \mathrm{~min}$ prior to starting permeation experiments. After rinsing off the solutions, the same volume of $145 \mathrm{mM}$ EN or $145 \mathrm{mM}$ NA in PBS was added to the donor chamber, and PBS in the receiver chamber was replaced. For inhibition of hydrolysis by skin esterase, DFP, a serine protease inhibitor, was added to the PBS in the receiver chamber at a concentration of $2.7 \mathrm{mM}$ during hydration and $0.54 \mathrm{mM}$ during permeation [17]. The donor and receiver solutions were stirred with a Teflon stirrer bar driven by a magnetic stirrer and maintained at $32^{\circ} \mathrm{C}$ throughout the experiments. An aliquot was sampled periodically from the receiver chamber, and the same volume of PBS or $0.54 \mathrm{mM}$ DFP in PBS was added to the chamber to keep the volume constant. The samples were mixed with the same volume of acetonitrile containing $1 \mathrm{mM}$ methyl 4-hydroxy benzoate as an internal standard for HPLC analysis, the mixture was centrifuged at $21,500 \mathrm{rpm}$ and $4^{\circ} \mathrm{C}$ for $5 \mathrm{~min}$, and the supernatant was stored at $4^{\circ} \mathrm{C}$ until determination of EN and NA concentrations. 


\section{Hydrolysis experiment in skin homogenate}

Rat skin was freshly excised by the same method as for the skin permeation experiment and homogenized directly with PBS at $4^{\circ} \mathrm{C}$ for 2 min using a homogenizer (Polytron PT 1200 E, Kinematica AG, Littau-Lucerne, Switzerland) to make $10 \%$ skin homogenate. The homogenate was centrifuged at $9,000 \times g$ and $4^{\circ} \mathrm{C}$ for $20 \mathrm{~min}$, and the supernatant, except for the floating lipid layers, was stored at $-80^{\circ} \mathrm{C}$ until the following experiments. The supernatant was thawed on ice and preincubated at $37^{\circ} \mathrm{C}$ for 10 min together with PBS containing EN at various concentrations. Two tubes of solutions were mixed, resulting in a final concentration of EN of 0.098-4.9 $\mathrm{mM}$ and in the skin homogenate of $0.2 \%$. The reaction solution was kept at $37^{\circ} \mathrm{C}$ and sampled at 0,5 , and $10 \mathrm{~min}$. The sample was mixed with the same volume of acetonitrile containing $16 \%$ trichloroacetic acid and $1 \mathrm{mM}$ methyl 4-hydroxy benzoate, the mixture was centrifuged $\left(21,500 \mathrm{rpm}, 4^{\circ} \mathrm{C}\right.$ and $\left.5 \mathrm{~min}\right)$, and the concentration of NA in the supernatant was determined. Protein content in the reaction solution was determined by Lowry's method [20].

\section{Determination of skin concentrations}

EN was applied for $6 \mathrm{~h}$ to $0.95 \mathrm{~cm}^{2}$ area of abdominal skin of hairless rat under anesthesia by i.p injection of the above anesthetic as $3.0 \mathrm{~mL}$ of $145 \mathrm{mM}$ EN in PBS. After removing and wiping EN solution, the applied area of skin was immediately excised and the excess subcutaneous fat was trimmed off. The skin sample was washed with $1 \mathrm{~mL}$ PBS three times, dipped into liquid nitrogen and then stored at $-80^{\circ} \mathrm{C}$ until the determination of EN and NA concentrations. After thawing the skin sample at $4^{\circ} \mathrm{C}$, it was dipped into $2.7 \mathrm{mM}$ DFP in PBS for $30 \mathrm{~min}$ to completely inhibit esterase activity. It was weighed, minced with scissors and homogenized with $0.5 \mathrm{~mL}$ PBS at $12,000 \mathrm{rpm}$ and $4^{\circ} \mathrm{C}$ for $5 \mathrm{~min}$. Acetonitrile $(0.5 \mathrm{~mL})$ containing $16 \%$ trichloroacetic acid for deproteinization and $1 \mathrm{mM}$ methyl 4-hydroxy benzoate was added to the skin homogenate, followed by agitation at $32^{\circ} \mathrm{C}$ for $15 \mathrm{~min}$, and then the mixture was centrifuged $\left(21,500 \mathrm{rpm}, 4^{\circ} \mathrm{C}\right.$ and $\left.5 \mathrm{~min}\right)$. The EN and NA concentrations in the resulting supernatant were determined by HPLC.

\section{High-performance liquid chromatography analysis}

The HPLC system (Shimadzu; Kyoto, Japan) consisted of a system controller (SCL-10AVP), pump (LC-20AD), autosampler (SIL-20A), column oven (CTO-20A), UV detector (SPD-20A), and analysis software (LC Solution). The column was Inertsil ${ }^{\circledR}$ ODS $-34.6 \mathrm{~mm} \times 250 \mathrm{~mm}$ (GL Sciences Inc.; Tokyo, Japan), which was maintained at $40^{\circ} \mathrm{C}$. The mobile phase was acetonitrile: $33.3 \mathrm{mM}$ phosphate buffer $(\mathrm{pH} 6.5)=20: 80$ containing $5.0 \mathrm{mM}$ dodecyltrimethylammonium chloride, and the flow rate was adjusted to $1.0 \mathrm{~mL} / \mathrm{min}$. EN and NA were detected at UV $260 \mathrm{~nm}$.

\section{Data analysis}

Figure 1 shows a three-layered diffusion model consisting of the stratum corneum, viable epidermis and dermis for the in

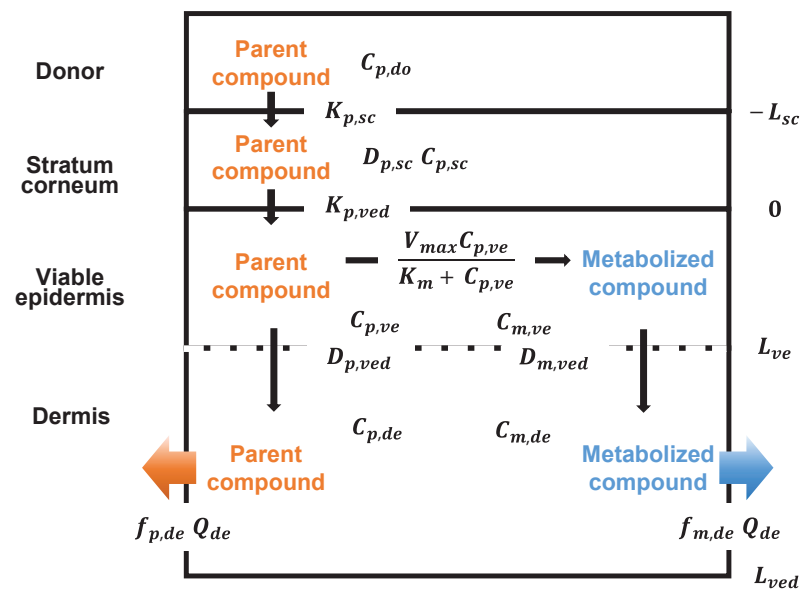

Figure 1: A three-layered diffusion model consisting of the stratum corneum, viable epidermis and dermis for the in vivo simultaneous skin transport and metabolism of chemical compounds. $C, K, D, L, V_{\text {max }}, K_{m}, Q$ and $f$ are the concentration, partition coefficient from the donor vehicle, diffusion coefficient, thickness, maximum metabolic rate, Michaelis constant, plasma volume flow and unbound fraction; the subscripts $p$ and $m$ refer to the parent compound and metabolite; and the subscripts do, sc, ve, de and denote the donor, stratum corneum, viable epidermis, dermis and both of viable epidermis and dermis, respectively.

vivo simultaneous skin transport and metabolism of chemical compounds. It was assumed that the stratum corneum is different from other lower layers in the diffusion and partition properties, and that these properties of viable epidermis and dermis are the same. Metabolic activity and blood clearance were supposed to take place only in the viable epidermis and dermis, respectively. According to Fick's second law of diffusion, the concentration of parent compound in the stratum corneum at position $x$ and time $t$ is given by

$$
\frac{\partial C_{p, s c}}{\partial t}=D_{p, s c} \frac{\partial^{2} C_{p, s c}}{\partial x^{2}}
$$

where $C$ and $D$ are the concentration and diffusion coefficients, and the subscripts $p$ and $s c$ refer to the parent compound and stratum corneum, respectively. In the viable epidermis where parent compound is transformed to metabolized compound, the rate of change in the concentrations of chemicals with time at a position are described as follows:

$$
\begin{gathered}
\frac{\partial C_{p, v e}}{\partial t}=D_{p, v e d} \frac{\partial^{2} C_{p, v e}}{\partial x^{2}}-\frac{V_{\max } C_{p, v e}}{K_{m}+C_{p, v e}} \\
\frac{\partial C_{m, v e}}{\partial t}=D_{m, v e d} \frac{\partial^{2} C_{m, v e}}{\partial x^{2}}+\frac{V_{\max } C_{p, v e}}{K_{m}+C_{p, v e}}
\end{gathered}
$$

where $V_{\max }$ and $K_{m}$ are the maximum metabolic rate and Michaelis constant, and the subscripts $m$, ve and ved refer to the metabolite, viable epidermis, and both of viable epidermis and dermis, respectively. Assuming remarkably higher concentrations of chemicals in the dermis than plasma, the concentration changes in the dermis are represented by

$$
\begin{aligned}
\frac{\partial C_{p, d e}}{\partial t} & =D_{p, v e d} \frac{\partial^{2} C_{p, d e}}{\partial x^{2}}-Q_{d e} f_{p, d e} C_{p, d e} \\
\frac{\partial C_{m, d e}}{\partial t} & =D_{m, v e d} \frac{\partial^{2} C_{m, d e}}{\partial x^{2}}-Q_{d e} f_{m, d e} C_{m, d e}
\end{aligned}
$$


where $Q$ and $f$ are the plasma volume flow and unbound fraction, and the subscript de denotes the dermis.

Initial and boundary conditions of the skin concentrations of two compounds are defined by:

$$
\begin{array}{ll}
-L_{s c}<x<0(t=0) & C_{p, s c}=0 \\
0<x<L_{v e}(t=0) & C_{p, v e}=C_{m, v e}=0 \\
L_{v e}<x<L_{v e d}(t=0) & C_{p, d e}=C_{m, d e}=0 \\
x=-L_{s c}(t>0) & C_{p, s c}=K_{p, s c} C_{d o} \\
x=0(t>0) & K_{p, s c} C_{p, v e}=K_{p, v e} C_{p, s c} \\
D_{p, s c} \frac{d C_{p, s c}}{d x}=D_{p, v e d} \frac{d C_{p, v e}}{d x} & \\
\frac{d C_{m, v e}=0}{d x} & \\
x=L_{v e}(t>0) & C_{p, v e}=C_{p, d e} \\
C_{m, v e}=C_{m, d e} & \\
x=L_{v e d}(t>0) & C_{p, d e}=C_{m, d e}=0
\end{array}
$$

where $L$ and $K$ are the thickness and partition coefficients from the donor vehicle, and the subscript $d o$ refers to donor solution, respectively. The concentration of parent and metabolized compounds at any position of the skin, $x$ at any time $t$, can be obtained by solving equations (1)-(5) under the conditions defined by equations (6)-(15).

The solution of these equations containing a non-linear term, i.e. Michaelis-Menten equation, is possible by numerical analysis using finite differences [21]. The right and left sides of Fick's second law of diffusion can be written approximately as:

$$
\begin{aligned}
& \frac{d C_{i, j}}{d t}=\frac{C_{i, j+1}-C_{i, j}}{\Delta t} \\
& \frac{d^{2} C_{i, j}}{d x^{2}}=\frac{C_{i-1, j}-2 C_{i, j}+C_{i+1, j}}{\Delta x^{2}}
\end{aligned}
$$

where $C_{i, j}$ is compound concentration in the $i$-th layer at $j$-th time, $\Delta x$ and $\Delta t$ are $x_{i+1}-x_{i}$ and $t_{j+1}-t_{j}$ respectively. By substituting equations (16) and (17) for equations (1)-(5) and arranging, we find:

$$
\begin{aligned}
& C_{p, s c i, j+1}=r D_{p, s c} C_{p, s c i-1, j}+\left(1-2 r D_{p, s c}\right) C_{p, s c i, j}+r D_{p, s c} C_{p, s c i+1, j} \\
& C_{p, v e i, j+1}=r D_{p, v e d} C_{p, v e i-1, j}+\left(1-2 r D_{p, v e d}\right) C_{p, v e i, j}+r D_{p, v e d} C_{p, v e i+1, j}-\Delta t \frac{V_{m a x} C_{p, v e i, j}}{K_{m}+C_{p, v e i, j}} \\
& C_{m, v e i, j+1}=r D_{m, v e d} C_{m, v e i-1, j}+\left(1-2 r D_{m, v e d}\right) C_{m, v e i, j}+r D_{m, v e d} C_{m, v e i+1, j}+\Delta t \frac{V_{\max } C_{p, v e i, j}}{K_{m}+C_{p, v e i, j}} \\
& C_{p, d e i, j+1}=r D_{p, v e d} C_{p, d e i-1, j}+\left(1-2 r D_{p, v e d}\right) C_{p, d e i, j}+r D_{p, v e d} C_{p, d e i+1, j}-\Delta t Q_{d e} f_{p, d e} C_{p, d e i, .} \\
& C_{m, d e i, j+1}=r D_{m, v e d} C_{m, d e i-1, j}+\left(1-2 r D_{m, v e d}\right) C_{m, d e i, j}+r D_{m, v e d} C_{m, d e i+1, j}-\Delta t Q_{d e} f_{m, d e} C_{m, d e}
\end{aligned}
$$

where $r=\Delta t / \Delta x^{2}$.
Skin permeation parameters, which were required to predict the skin concentrations of the parent compound and its metabolite, were obtained by fitting permeation data under various conditions to the diffusion models [22]. In order to obtain the $D_{m \text {, ved }}$ value, the permeation data for NA applied to stripped skin were fitted to the one-layered diffusion model. The same model was used to determine $D_{p \text {, ved }}$ and $K_{p \text {, ve }}$ values from the permeation data of EN applied to stripped skin during DFP treatment. $D_{p, s c}$ and $K_{p, s c}$ values were estimated by fitting the permeation data of EN through the DFP-treated full-thickness skin to the two-layered diffusion model using the previously obtained values of $D_{p, \text { ved }}$ and $K_{p, v e}$. Hydrolysis profiles of the ester in the skin homogenate were fitted to the Michaelis-Menten equation and then the skin metabolic parameters, $V_{\max }$ and $K_{m}$, were determined. A weighted leastsquared method based on a quasi-Newton algorithm, which was run on the solver-function of Microsoft Excel 2016, was used to estimate the skin permeation and metabolic parameters. Skin concentrations of both the parent compound and its metabolite at various distances from skin surface were calculated from equations (18)-(22), and the volume-mean concentrations were obtained from these data. In the calculation, the skin permeation and metabolic parameters were fixed as the values obtained above, and the values of thickness of each layer ( $L_{s c}$ , $L_{v e}$ and $\left.L_{v e d}\right)$, plasma flow rate $\left(Q_{d e}\right)$ and unbound fraction $\left(f_{p, d e}\right.$ and $\left.f_{m, d e}\right)$ were taken from the literatures [11, 23, 24]. $V_{\max }$ was set to an eleven times higher value, because the enzyme distribution only in the viable epidermis was assumed and thickness of the layer was about one-eleventh of whole skin. When a homogeneous enzyme distribution in the viable epidermis and dermis was assumed, a Michaelis-Menten term $\left(-\triangle t V_{\max } C_{p, d e i, j} / K_{m}+C_{p, d e i, j}\right.$ and $\quad+\triangle t V_{\max } C_{p, d e i, j} / K_{m}+C_{p, d e i, j}$ ) was introduced into equations (21) and (22), and the $V_{\max }$ values obtained from the hydrolysis experiment in skin homogenate was just used. When influence of plasma clearance upon the estimated skin concentration was taken into account, $-\triangle t Q_{d e} f_{p, d e} C_{p, d e i, j}$ and $-\triangle t Q_{d e} f_{m, d e} C_{m, d e i, j}$ were removed from equations (21) and (22).

\section{Results}

\section{Determination of skin permeation parameters}

Figure 2a shows the cumulative amount of NA permeated through the tape-stripped skin after application of NA solution. The cumulative amount increased with time after a short lag time, and the behavior was described by the one-layered diffusion model. The obtained values of the permeation parameters, $D_{m, v e d}$ and $K_{m, v e}$, are listed in Table 1. NA permeation through the full-thickness skin was analyzed by the two-layered diffusion model using the previously obtained $D_{m \text {, ved }}$ and $K_{m, v e}$ values as shown in Figure $2 \mathrm{~b}$, and the parameters relating to the NA permeation through the stratum corneum, $D_{m, s c}$ and $K_{m, s c}$ were calculated as listed in Table 1. The permeation parameters of EN, $D_{p, v e d}, D_{p, s c}, K_{p, v e}$ and $K_{p, s c}$, in Table 1 were determined from the permeation data after application EN to the skin treated with DFP, a serine protease inhibitor (Figures $2 \mathrm{c}$ and $\mathrm{d}$ ). The permeation profiles 
of EN through the stripped skin and full-thickness skin could also be described by the one-layered and two-layered diffusion models, respectively. Almost no flux of NA was observed after DFP treatment.

\section{Determination of skin metabolic parameters}

Figure 3 shows the relation between the hydrolysis rate of EN to NA and initial EN concentration in $0.2 \%$ skin homogenate. The hydrolysis of EN followed Michaelis-Menten kinetics, and the kinetic parameters ( $V_{\max }$ and $K_{m}$ ) estimated by data-fitting are listed in Table 1.

\section{Estimation of skin concentrations}

In vivo skin concentrations of both $\mathrm{NA}$ and $\mathrm{EN}$ after application of EN to skin surface without DFP treatment were estimated from equations (18)-(22) using the parameter values listed in Table 1. Additional three kinds of permeation models being different in the cutaneous enzyme distribution and plasma clearance were used in the estimation: homogeneous distribution model in the viable epidermis and dermis, homogeneous distribution model with plasma clearance in the dermis, and heterogeneous distribution model only in viable epidermis. Figure 4a shows the time courses of volume-mean concentrations of EN and NA, which were estimated assuming homogeneous enzyme distribution in the viable epidermis and dermis and assuming no plasma clearance. EN concentration sharply rose until $30 \mathrm{~min}$ after the skin application and slowly fell, and the value at $6 \mathrm{~h}$ was almost equal to the observed value. However, NA concentration increased much slower than EN concentration, and the value at $6 \mathrm{~h}$ was remarkably lower than the observed value. Plasma clearance lowered concentrations
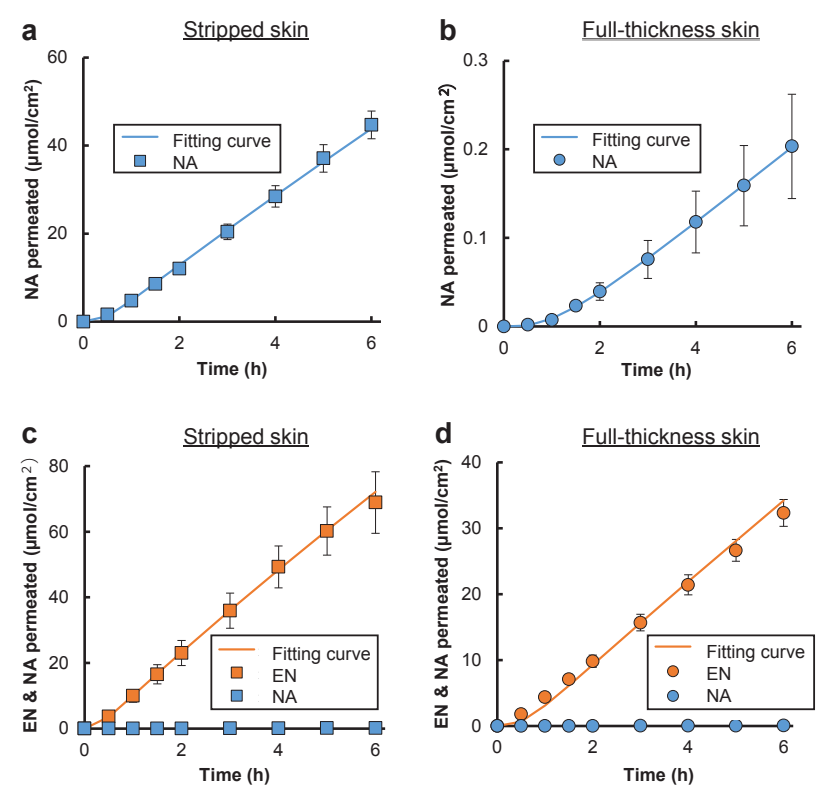

Figure 2: Skin permeation profiles of nicotinic acid (NA) and ethyl nicotinate (EN) through stripped skin $(a, c)$ and full-thickness skin $(b, d)$ from NA solution $(a, b)$ and EN solution with diisopropyl fluorophosphate (DFP) (c, d). Blue and orange symbols are NA and EN, and squares and circles refer to stripped skin and full-thickness skin. Each symbol represents the mean \pm S.E. of 3 (b) and 4 experiments ( $a, c$ and d). Solid curves show the calculated data best-fitted to the one-layered $(a, b)$ and two-layered (c, d) diffusion models.
Table 1: Parameter values used in the estimation of skin concentration of a dermally metabolized chemical, ethyl nicotinate (EN), and its metabolite, nicotinic acid (NA). ${ }^{\text {a }}$

\begin{tabular}{|c|c|c|}
\hline Parameter & Description & Value \\
\hline$D_{m, v e d}$ & $\begin{array}{l}\text { Diffusion coefficient of the metabolite in } \\
\text { the viable epidermis and dermis }\end{array}$ & $1.31 \times 10^{-3} \pm 0.16 \times 10^{-3}\left(\mathrm{~cm}^{2} / \mathrm{h}\right)$ \\
\hline$K_{m, v e}$ & $\begin{array}{l}\text { Partition coefficient of the metabolite } \\
\text { from the vehicle to the viable epidermis }\end{array}$ & $2.62 \pm 0.18$ \\
\hline$D_{m, s c}$ & $\begin{array}{l}\text { Diffusion coefficient of the metabolite in } \\
\text { the stratum corneum }\end{array}$ & $2.74 \times 10^{-6} \pm 0.43 \times 10^{-6}\left(\mathrm{~cm}^{2} / \mathrm{h}\right)$ \\
\hline$K_{m, s c}$ & $\begin{array}{l}\text { Partition coefficient of the metabolite } \\
\text { from the vehicle to the stratum corneum }\end{array}$ & $0.165 \pm 0.025$ \\
\hline$D_{p, \text { ved }}$ & $\begin{array}{c}\text { Diffusion coefficient of the parent } \\
\text { compound in the viable epidermis and } \\
\text { dermis }\end{array}$ & $2.38 \times 10^{-3} \pm 0.42 \times 10^{-3}\left(\mathrm{~cm}^{2} / \mathrm{h}\right)$ \\
\hline$K_{p, v e}$ & $\begin{array}{c}\text { Partition coefficient of the parent } \\
\text { compound from the vehicle to the viable } \\
\text { epidermis }\end{array}$ & $2.35 \pm 0.12$ \\
\hline$D_{p, s c}$ & $\begin{array}{l}\text { Diffusion coefficient of the parent } \\
\text { compound in the stratum corneum }\end{array}$ & $7.44 \times 10^{-6} \pm 2.23 \times 10^{-6}\left(\mathrm{~cm}^{2} / \mathrm{h}\right)$ \\
\hline$K_{p, s c}$ & $\begin{array}{l}\text { Partition coefficient of the parent } \\
\text { compound from the vehicle to the } \\
\text { stratum corneum }\end{array}$ & $23.5 \pm 16.7$ \\
\hline$L_{v e d}$ & $\begin{array}{l}\text { Thickness of the viable epidermis and } \\
\text { dermis }\end{array}$ & $585(\mu \mathrm{m})^{\mathrm{b}}$ \\
\hline$L_{v e}$ & $\begin{array}{c}\text { Thickness of the viable epidermis and } \\
\text { dermis }\end{array}$ & $53(\mu \mathrm{m})^{c}$ \\
\hline$L_{s c}$ & Thickness of the stratum corneum & $15(\mu \mathrm{m})^{\mathrm{b}}$ \\
\hline$V_{\max }$ & Maximum metabolic rate & $\begin{array}{c}7.06 \times 10^{-2} \pm 0.93 \times 10^{-2} \\
(\mu \mathrm{mol} / \mathrm{min} / \mathrm{mg} \text { of protein) }\end{array}$ \\
\hline$K_{m}$ & Michaelis constant & $1.73 \pm 0.407(\mathrm{mM})$ \\
\hline$Q_{d e}$ & Plasma volume flow in the dermis & $3.71(\mathrm{~mL} / \mathrm{h} / \mathrm{g})^{\mathrm{d}}$ \\
\hline$f_{p, d e}$ & $\begin{array}{l}\text { Unbound fraction of the parent } \\
\text { compound in the dermis }\end{array}$ & 1.0 \\
\hline$f_{m, d e}$ & $\begin{array}{l}\text { Unbound fraction of the metabolite in } \\
\text { the dermis }\end{array}$ & 1.0 \\
\hline \multicolumn{3}{|c|}{$\begin{array}{l}\text { aEach value represents the mean } \pm \text { S.E. of 3-4 experime } \\
\text { bValue was cited from the literature [11]. } \\
\text { cValue was cited from the literature [23]. } \\
\text { dValue was cited from the literature [24]. }\end{array}$} \\
\hline
\end{tabular}

of both EN and NA, and the effect on NA was larger than EN, as shown in Figure $4 \mathrm{~b}$. NA concentration was overestimated by assuming enzyme distribution only in the viable epidermis without plasma clearance, although little change of EN profile was observed in Figure 4c. Both EN and NA concentrations could be estimated to be values corresponding to the observed values only just by three-layered diffusion model considering both heterogeneous enzyme distribution and plasma clearance (Figure 4d).

Figure 5a shows the time-dependent changes in the concentration-distance profiles of EN in the full-thickness skin, which were estimated by the improved three-layered diffusion model. The enlarged profiles of both the viable epidermis/dermis and stratum corneum regions in the EN profiles are also shown in Figures $5 b, c$, because of the large 


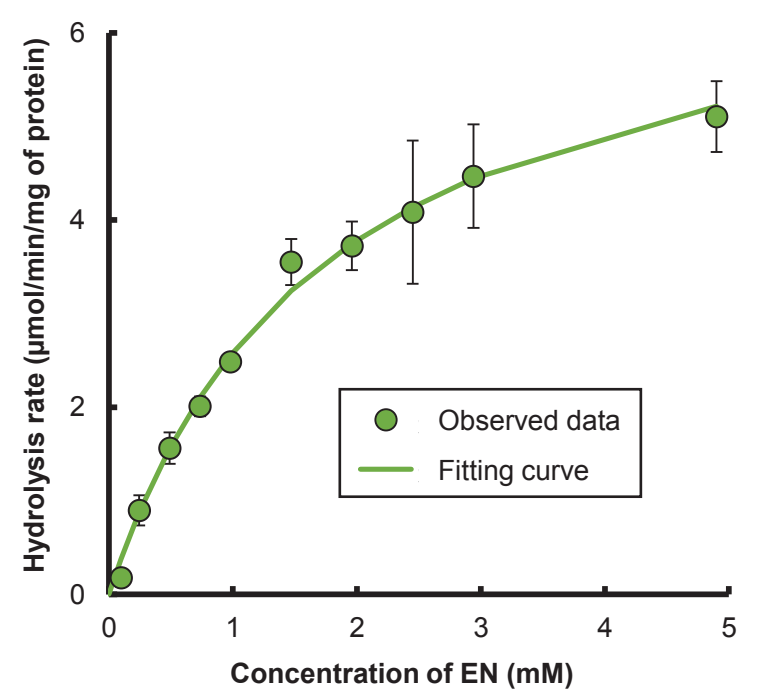

Figure 3: Hydrolysis profiles of ethyl nicotinate (EN) to nicotinic acid (NA) at various initial concentrations of EN in $0.2 \%$ skin homogenate. Each symbol represents the mean \pm S.E. of 3 experiments. The solid curve shows the calculated data best-fitted to the Michaelis-Menten equation. difference in EN concentration between the two regions. EN concentration in the stratum corneum at the interface with the viable epidermis increased with time until about $30 \mathrm{~min}$, resulting in a linear concentration gradient inside the stratum corneum, and then the concentration at the skin surface side decreased gradually (Figure 5c). Although increasing and decreasing EN concentrations in the viable epidermis at the interface with the stratum corneum depended on the change in concentration in the stratum corneum, all concentrationdistance profiles in the viable epidermis and dermis described biphasic concave curves during the experimental period: a rapid decline in the viable epidermis and a slow decline in the dermis (Figure 5b). In contrast, the concentration-distance profiles of NA showed convex curves in the viable epidermis followed by concave curves in the dermis as shown in Figure $5 \mathrm{~d}$. The concentration of NA was raised with time until $1 \mathrm{~h}$ and remained unchanged until $6 \mathrm{~h}$.

In order to more precisely assess the influence of cutaneous enzyme distribution and plasma clearance on

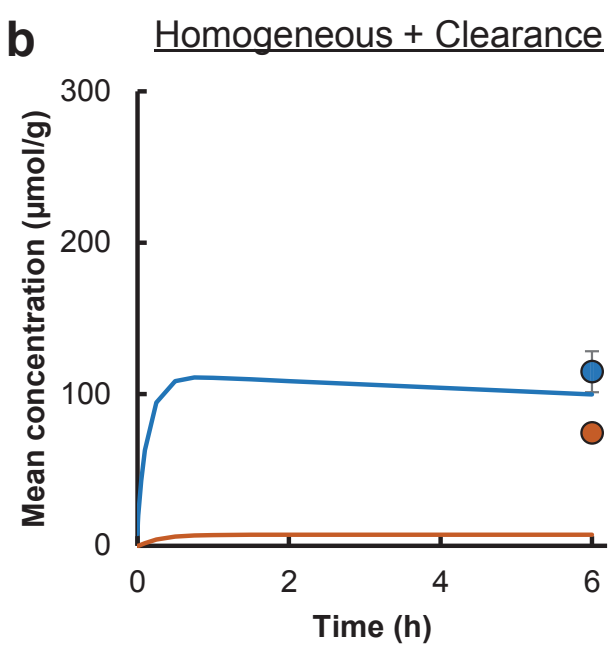

d Heterogeneous + Clearance
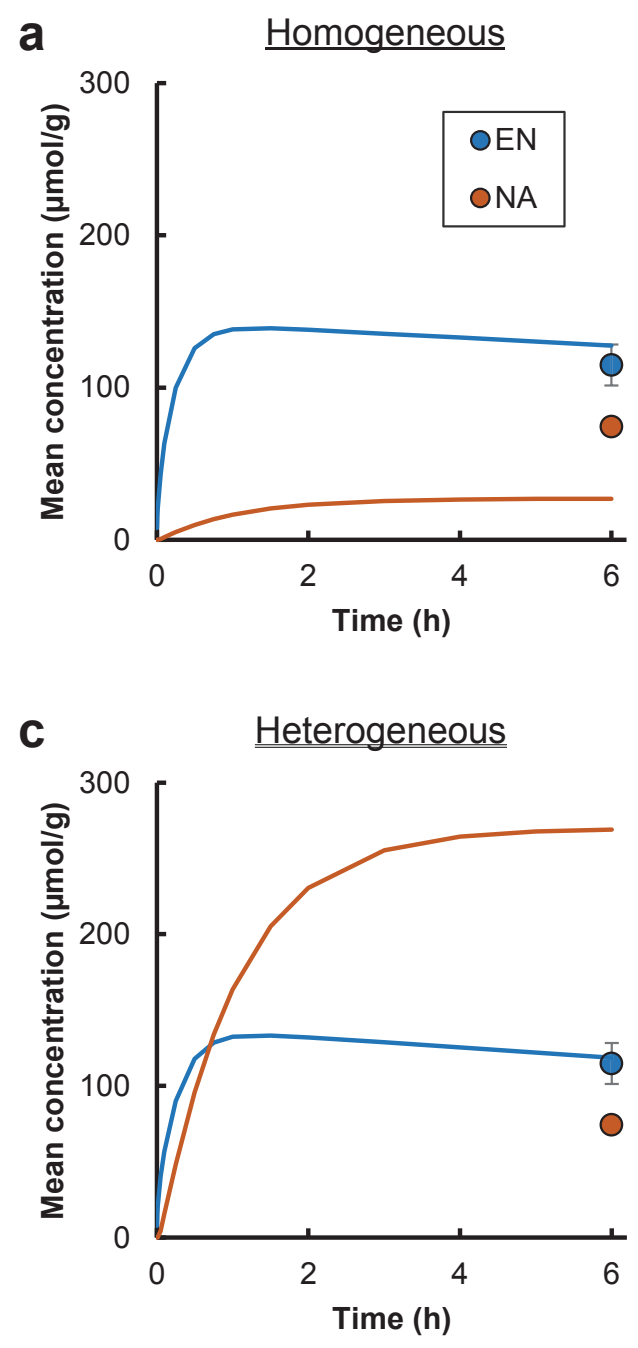

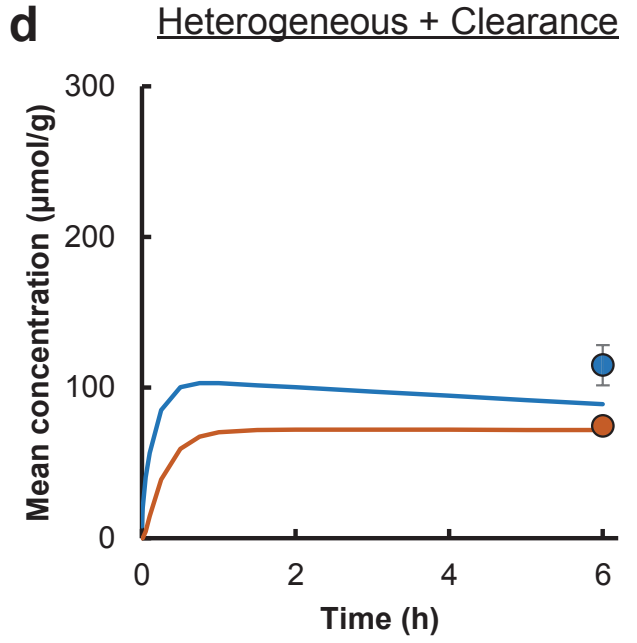


the concentration of EN and NA, the concentration-distance profiles were simulated based on additional three permeation models. Because the profiles of EN in the stratum corneum were not so different among models, the profiles of both EN and NA in the viable epidermis and dermis are shown in Figure 6. Homogeneous enzyme distribution in the viable epidermis and dermis caused a monolithic decrease of EN concentration with an increase of distance from skin surface (Figure 6a,b), whereas enzyme distribution only in the viable epidermis produced a biphasic decline (Figures 5b,6c). Existence of plasma clearance reduced EN concentrations throughout the profiles (Figures 5b,6b). NA concentration was raised to be about 10 times by heterogeneous enzyme distribution, although the shapes of NA concentration-distance profiles were not changed (Figures 5d,6f). The concentration was reduced by plasma clearance (Figures 5d,6e), and the effect was higher than EN concentration.

\section{Discussion}

We previously proposed a two-layered diffusion model to estimate the mean skin concentration and concentration at each position in the skin layers [11, 12]. However, the use of this model may result in an inaccurate estimation of skin concentration for chemicals extensively metabolized in the skin. Because a large part of steroids contained in the topical formulations are ante-drugs [14] and because transdermal drug delivery via prodrug derivation has been much attempted [15], further improvement of our method is desirable for expanding to enable the estimation of skin concentrations after dermal exposure of chemicals extensively metabolized in the skin. The skin, especially epidermis, is metabolically active [13], and any permeant is subjected to the metabolic properties of the living layer. Moreover, both applied chemical compound and its metabolite are cleared by blood microcirculation of permeable capillaries in the dermis after passing through the viable epidermis [16]. A three-layered diffusion model was then constructed by incorporating the Michaelis-Menten equation and plasma clearance into the viable epidermis and dermis, respectively (Figure 1). In our strategy, the values of skin permeation and metabolic parameters were obtained from separate in vitro experiments (Figures 2,3) and these were introduced into equations (18)-(22), which were derived from the three-layered diffusion model. The calculated mean skin concentrations of both EN and NA after EN application were similar with the values observed in vivo (Figure 4).

Influence of cutaneous enzyme distribution and plasma clearance on the skin concentration of EN and NA was assessed by the simulation of mean concentration and concentrationdistance profiles based on additional three permeation models (Figures 4-6). The simulation results suggested that the cutaneous esterases are localized in the viable epidermis. A higher esterase activity was observed in the epidermal cells

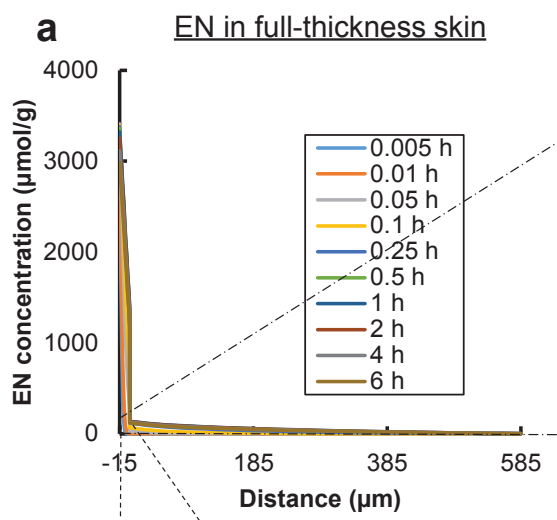

b EN in viable epidermis/dermis
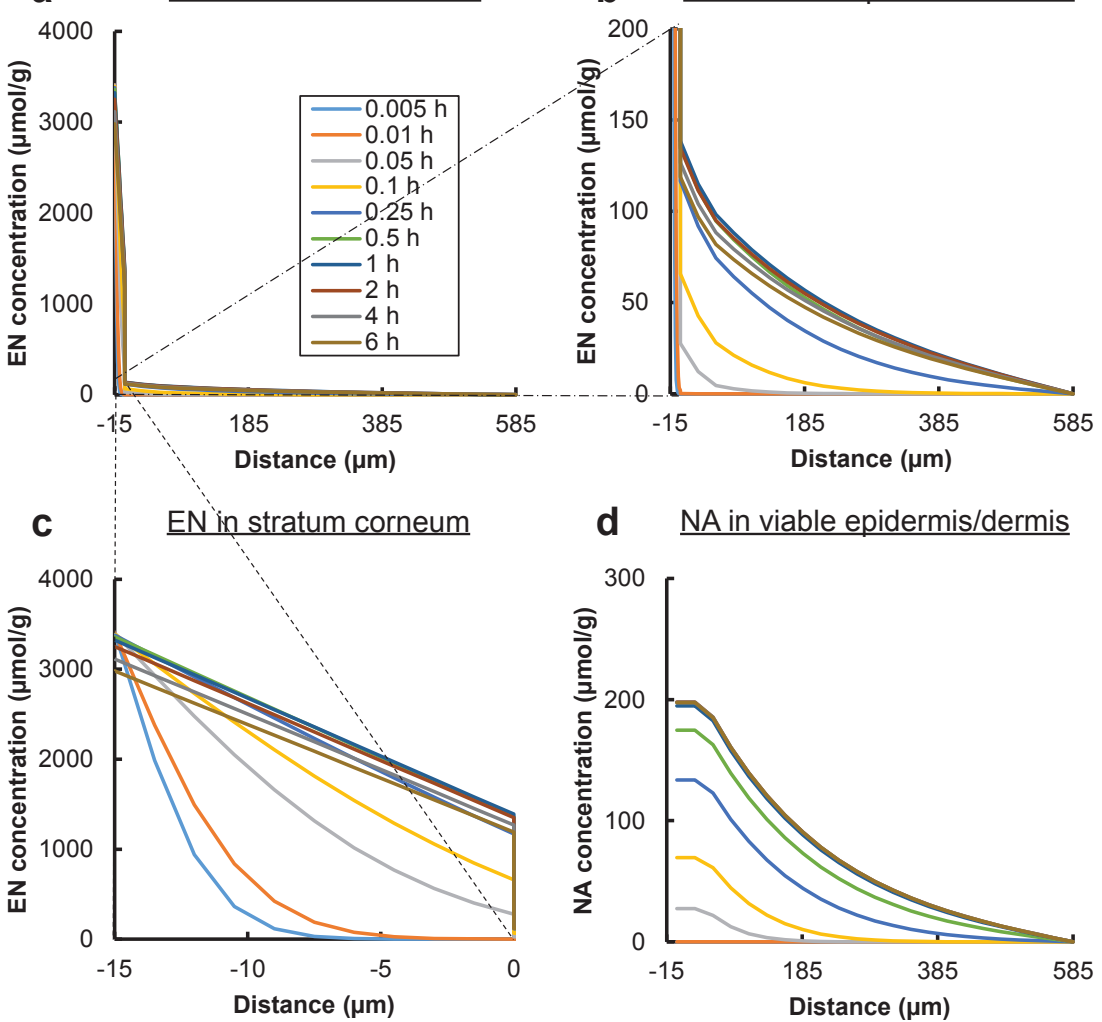

d $\quad$ NA in viable epidermis/dermis

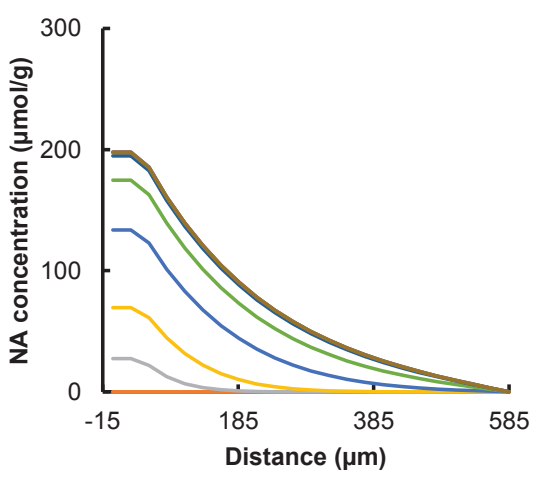


and near hair follicles than the dermis by a microphotographic study using fluorescein-5-isothiocyanate diacetate [25]. In contrast, an immunohistological study showed that nonspecific $\alpha$-naphthylacetate-esterase, by which nicotinates are metabolized to nicotinic acid, was also located in the dermis [26]. Carboxylesterases are present in cytosol as well as microsomal fractions of tissues [27], so that such dissolved enzymes may be washed out during histological preparation. The concentration of NA was more affected by the cutaneous enzyme distribution than that of EN. It is necessary to construct the skin permeation model accompanying an accurate enzyme location to estimate the skin concentration of metabolites.

The high metabolizing potential in the viable epidermis was attributed by the fact that barrier lipids in the stratum corneum are synthesized in the living layer together with keratinocyte differentiation [28]. The barrier lipids, their precursors and metabolizing enzymes are packaged into the epidermal lamellar body in the stratum spinosum and stratum granulosum, and are secreted at the interface between the stratum granulosum and stratum corneum by fusing the lamellar body with the apical plasma membrane of the outermost stratum granulosum cell layer [29]. The secreted enzymes include many lysosomal hydrolases [30], and the esterases present in the cytosol fraction of the skin were mainly responsible for the hydrolysis of propranolol prodrug [31]. Thus, some of the lysosomal enzymes must contribute to the metabolism of EN to NA in the skin. Carboxylesterases have a wide substrate specificity and play an important role in the hydrolytic biotransformation of many chemicals [32]. About $70 \%$ of the hydrolysis activity of prednisolone 21-acetate in skin homogenate from hairless mice were not deactivated by 3,4-dichloroisocoumarin, which completely inhibited the activity of carboxylesterase [33]. Hydrolysis of EN to NA must be mediated by many enzymes in the skin.

Two assumptions were given when plasma clearance was incorporated in the skin permeation model. One assumption is that the concentrations of both parent and metabolized chemicals in the plasma are negligible low compared with those in the dermis. In fact, indomethacin concentrations in the underlying tissues was 1000-10000 fold higher than those in the plasma following topical application [24]. Another is that the unbound fractions of both chemicals in the dermis are supposed to be 1.0. It may not be true even if these concentrations in the dermis are so high as to cause the saturation of tissue binding. The unbound fraction to the plasma protein can be estimated by a quantitative structureproperty relationship [34]. Such values should be used as an alternative to the fraction of unbound chemicals in the dermis. Skin microvasculature and its distribution affect cutaneous concentration profiles of topically applied chemicals [16]. The plasma clearance also affected the skin concentrations of EN and NA (Figures 4-6), and thus the effect must be taken care into the estimation of in vivo skin concentration from in vitro data.

EN concentration was harder affected than NA concentration. It would be caused by the deposit of EN in stratum corneum, because EN concentration in the stratum corneum was remarkably higher than that in the viable epidermis and dermis,

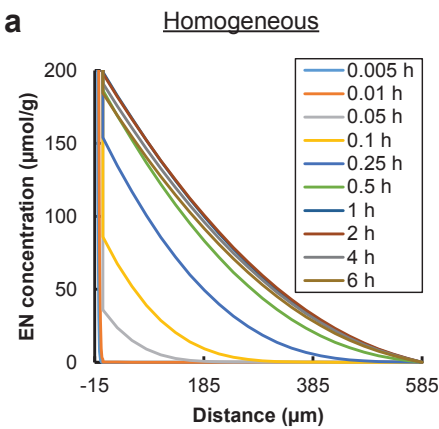

d

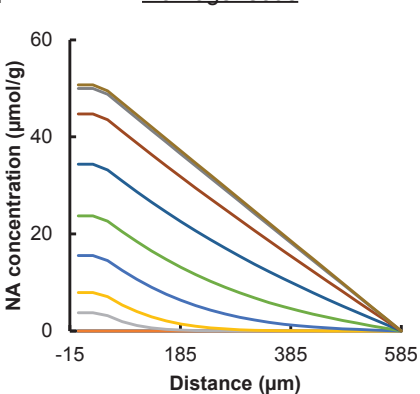

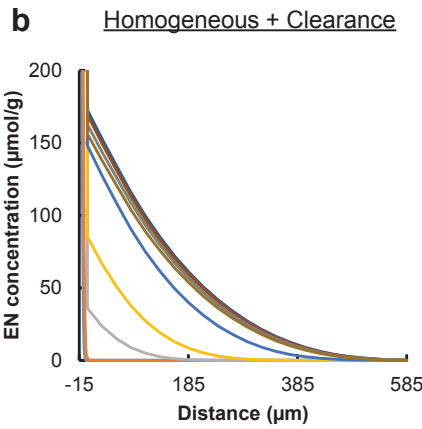

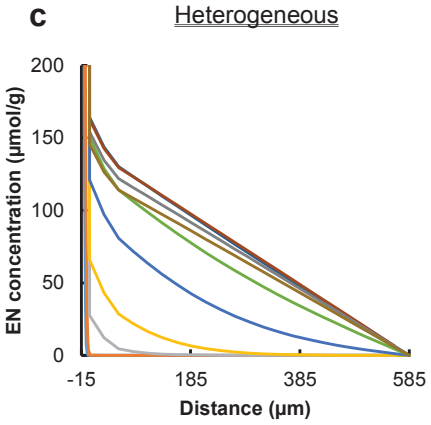

e Homogeneous + Clearance
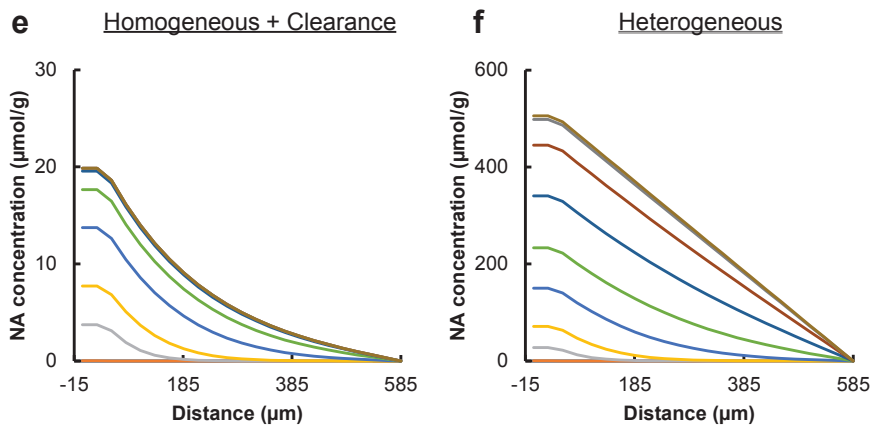
and EN concentration-distance profiles in stratum coneum were almost the same among the skin permeation models. Recently, in silico prediction of transdermal and systemic drug disposition by a physiologically based pharmacokinetic model, in which the stratum corneum was characterized by a brickand-mortar structure, was proposed $[7,35]$. The method is superior to overcome the numerical complexity of model by utilizing the quantitative structure-property relationships. Although the final goal of our skin permeation model is also a physiological model incorporating skin metabolism, a high predictability for the concentrations of extensively metabolized chemicals in skin was obtained at the present stage.

The in vivo skin concentrations of both EN and NA after EN application could be estimated by our improved model, a three-layered diffusion model based on the Fick's second law of diffusion, and incorporated Michaelis-Menten equation and plasma clearance term. Although skin permeation and metabolic parameters were obtained from in vitro skin permeation experiments in the present study, these parameters can be also predicted using the physicochemical properties of chemicals [36-38]. Only metabolic parameters that are determined from the distinct experiments are needed. There are many skin models, e.g. subcellular fractions of skin, tissue-cultured skin, monolayer cultures of keratinocytes or fibroblasts, and reconstructed human skin equivalents, other than skin homogenates and excised skin for assessing skin metabolism $[39,40]$. It is desirable to develop a simple model which mimicks accurate in vivo skin metabolism.

\section{Conclusion}

We proposed a three-layered diffusion model consisting of stratum corneum, viable epidermis and dermis, which was constructed based on Fick's second law of diffusion incorporated with Michaelis-Menten equation and plasma clearance in the viable epidermis and dermis, respectively. The skin concentrations calculated from our improved model using the permeation and metabolic parameters obtained beforehand were similar to the observed values. Influence of cutaneous enzyme distribution and plasma clearance on the skin concentrations were also estimated using appropriately modified models, resulting in higher influence on the acid than the ester. Our in silico estimation method of skin concentration of chemical and its metabolite gives important information about their efficacy and safety, and thus becomes a powerful tool to promote the development of pharmaceutical and cosmeceutical products.

\section{References}

1. Chang RK, Raw A, Lionberger R, Yu L (2013) Generic development of topical dermatologic products: formulation development, process development, and testing of topical dermatologic products. AAPS J. 15: 41-52. Link: https://goo.gl/nE91Qs

2. Blount BC, Milgram KE, Silva MJ, Malek NA, Reidy JA, et al. (2000) Quantitative detection of eight phthalate metabolites in human urine using HPLC-APCI-MS/ MS. Anal Chem 72: 4127-4134. Link: https://goo.gl/ZwbqZD

3. Rougier A, Dupuis D, Lotte C, Roguet R, Schaefer H (1983) In vivo correlation between stratum corneum reservoir function and percutaneous absorption. $J$ Invest Dermatol 81: 275-278. Link: https://goo.gl/3zjPsP
4. Groth L, Serup J (1998) Cutaneous microdialysis in man: effects of needle insertion trauma and anaesthesia on skin perfusion, erythema and skin thickness. Acta Derm Venereol 78: 5-9. Link: https://goo.gl/ysgxza

5. Lademann J, Meinke MC, Schanzer S, Richter H, Darvin ME, et al. (2012) In vivo methods for the analysis of the penetration of topically applied substances in and through the skin barrier. Int J Cosmet Sci 34: 551-559. Link: https://goo. $\mathrm{gl} / \mathrm{TkF3Pf}$

6. Selzer D, Neumann D, Neumann H, Kostka KH, Lehr CM, et al. (2015) A strategy for in-silico prediction of skin absorption in man. Eur J Pharm Biopharm 95: 6876. Link: https://goo.gl/5tymYl

7. Chen L, Han L, Saib O, Lian G (2015) In silico prediction of percutaneous absorption and disposition kinetics of chemicals. Pharm Res 32: 1779-1793. Link: https://goo.gl/zRalqx

8. Haftek M, Callejon S, Sandjeu Y, Padois K, Falson F, et al. (2011) Compartmentalization of the human stratum corneum by persistent tight junction-like structures. Exp Dermatol 20:617-621. Link: https:// goo.gl/lwgorC

9. Heisig M, Lieckfeldt R, Wittum G, Mazurkevich G, Lee G (1996) Non steady-state descriptions of drug permeation through stratum corneum. I. The biphasic brick-and-mortar model. Pharm Res. 13: 421-426. Link: goo.gl/4KaA2e

10. Blank IH (1965) Cutaneous barriers. J Invest Dermatol 45: 249-256. Link: https://goo.gl/mqCPIV

11. Oshizaka T, Kikuchi K, Kadhum WR, Todo H, Hatanaka T, et al. (2014) Estimation of skin concentrations of topically applied lidocaine at each depth profile. Int $J$ Pharm 475: 292-297. Link: https://goo.gl/HJV2w5

12. Hatanaka T, Yoshida S, Kadhum WR, Todo H, Sugibayashi K (2015) In Silico Estimation of Skin Concentration Following the Dermal Exposure to Chemicals. Pharm Res 32: 3965-3974. Link: https://goo.gl/tTXcQE

13. Kao J, Carver MP (1990) Cutaneous metabolism of xenobiotics. Drug Metab Rev 22: 363-410. Link: https://goo.gl/Uj2t7Y

14. Lee HJ, Cooperwood JS, You Z, Ko DH (2002) Prodrug and antedrug: two diametrical approaches in designing safer drugs. Arch Pharm Res 25: 111-136. Link: https://goo.gl/cMc3hc

15. Sloan KB (1992) Prodrugs, topical and ocular drug delivery, Marcel Dekker, New York

16. Cevc G, Vierl U (2007) Spatial distribution of cutaneous microvasculature and local drug clearance after drug application on the skin. J Control Release 118 18-26. Link: https://goo.gl/NRhh3A

17. Sugibayashi K, Hayashi T, Hatanaka T, Ogihara M, Morimoto Y (1996) Analysis of simultaneous transport and metabolism of ethyl nicotinate in hairless rat skin. Pharm Res 13: 855-860. Link: https://goo.gl/HfG3Ac

18. Rittirod T, Hatanaka T, Uraki A, Hino K, Katayama K, et al. (1999) Species difference in simultaneous transport and metabolism of ethyl nicotinate in skin Int J Pharm 178: 161-169. Link: https://goo.gl/3qFyqh

19. Flynn GL, Dürrheim H, Higuchi WI (1981) Permeation of hairless mouse skin II: membrane sectioning techniques and influence on alkanol permeabilities. $J$ Pharm Sci 70: 52-56. Link: https://goo.gl/zFt1xT

20. Lowry OH, Rosebrough NJ, Farr AL, Randall RJ (1951) Protein measurement with the Folin phenol reagent. J Biol Chem 193: 265-275. Link: https://goo.gl/gkPBZk

21. Higuchi WI, Gordon NA, Fox JL, Ho NFH (1983) Transdermal delivery of prodrugs. Drug Dev Ind Pharm 9: 691-706. Link: https://goo.gl/5kDHbp

22. Ohmori S, Hayashi T, Kawase M, Saito S, Sugibayashi K, et al. (2000) Transdermal delivery of the potent analgesic dihydroetorphine: kinetic analysis 
of skin permeation and analgesic effect in the hairless rat. J Pharm Pharmaco 52: 1437-1449. Link: https://goo.gl/v5jWCZ

23. Bronaugh RL, Stewart RF (1986) Methods for in vitro percutaneous absorption studies. VI: Preparation of the barrier layer. J Pharm Sci 75: 487-491. Link: https://goo.gl/Rvf3vX

24. Hatanaka T, Manabe E, Ohtsuki T, Okuyama K, Mori M, et al. (2000) Drug targeting efficacy to underlying muscle following topical application. I. Evaluation based on a physiological pharmacokinetic model. Biol Pharm Bull 23: 860-865. Link: https://goo.gl/cYp2wW

25. Sugibayashi K, Hayashi T, Morimoto Y (1999) Simultaneous transport and metabolism of ethyl nicotinate in hairless rat skin after its topical application: the effect of enzyme distribution in skin. J Control Release 62: 201-208. Link: https://goo.gl/BLU73B

26. Müller B, Kasper M, Surber C, Imanidis G (2003) Permeation, metabolism and site of action concentration of nicotinic acid derivatives in human skin Correlation with topical pharmacological effect. Eur J Pharm Sci 20: 181-195. Link: https://goo.gl/fXRIKP

27. Jewell C, Prusakiewicz JJ, Ackermann C, Payne NA, Fate G, et al. (2007) Hydrolysis of a series of parabens by skin microsomes and cytosol from human and minipigs and in whole skin in short-term culture. Toxicol App Pharmacol 225: 221-228. Link: https://goo.gl/TjIxDK

28. Breiden B, Sandhoff K (2014) The role of sphingolipid metabolism in cutaneous permeability barrier formation. Biochim Biophys Acta 1841: 441-452. Link: https://goo.gl/r3XXfv

29. Uchida Y, Holleran WM (2008) Omega-O-acylceramide, a lipid essential for mammalian survival. J Dermatol Sci 51: 77-87. Link: https://goo.gl/FMGBiS

30. Grayson S, Johnson-Winegar AG, Wintroub BU, Isseroff RR, Epstein EH Jr., et al. (1985) Lamellar body-enriched fractions from neonatal mice: preparative techniques and partial characterization. J Invest Dermatol 85: 289-294. Link: https://goo.gl/vE1JNV
31. Ahmed S, Imai T, Otagiri M (1995) Stereoselective hydrolysis and penetration of propranolol prodrugs: in vitro evaluation using hairless mouse skin. J Pharm Sci 84: 877-883. Link: https://goo.gl/QgfZJf

32. Satoh T, Hosokawa M (2006) Structure, function and regulation of carboxylesterases. Chem Biol Interact 162: 195-211 Link: https://goo.gl/UnFmPQ

33. Hikima T, Ohno Y, Maibach HI (2001) Metabolism of prednisolone 21-acetate in hairless mouse skin. Skin Pharmacol. Appl Skin Physiol 14: 203-209. Link: https://goo.gl/Zlzy1v

34. Yamazaki K, Kanaoka M (2004) Computational prediction of the plasma protein-binding percent of diverse pharmaceutical compounds. J Pharm Sci 93: 1480-1494. Link: https://goo.gl/DXgFs4

35. Chen T, Lian G, Kattou P (2016) In silico modelling of transdermal and systemic kinetics of topically applied solutes: Model development and initial validation for transdermal nicotine. Pharm Res 33: 1602-1614. Link: t. hs:/ goo.gl/4lhaO1

36. Hatanaka T, Inuma M, Sugibayashi K, Morimoto Y (1990) Prediction of skin permeability of drugs. I. Comparison with artificial membrane. Chem Pharm Bull 38: 3452-3459. Link: https://goo.gl/bSRTJY

37. Potts RO, Guy RH (1992) Predicting skin permeability. Pharm Res 9: 663-669 Link: https://goo.gl/XC6crM

38. Geinoz S, Guy RH, Testa B, Carrupt PA (2004) Quantitative structure-permeation relationships (QSPeRs) to predict skin permeation: a critical evaluation. Pharm Res 21: 83-92. Link: https://goo.gl/pb7qt

39. Borchardt RT, Smith PL (1996) Models for assessing drug absorption and metabolism, Plenum Press, New York.

40. Wiegand C, Hewitt NJ, Merk HF, Reisinger K (2014) Dermal xenobiotic metabolism: A comparison between native human skin, four in vitro skin test systems and a liver system. Skin Pharmacol Physiol 27: 263-275.. Link: https:// goo.gl/XIhhJg

Copyright: (c) 2017 Hatanaka T, et al. This is an open-access article distributed under the terms of the Creative Commons Attribution License, which permits unrestricted use, distribution, and $r$ eproduction in any medium, provided the original author and source are credited. 